\title{
Synthetic opportunities and challenges for macrocyclic kinase inhibitors
}

Jennifer Alisa Amrhein ${ }^{1,2}$, Stefan Knapp ${ }^{1,2}{ }^{*}$, Thomas Hanke ${ }^{1,2}$ *

${ }^{1}$ Institute for Pharmaceutical Chemistry, Johann Wolfgang Goethe-University, Max-von-LaueStr. 9, D-60438 Frankfurt am Main, Germany.

${ }^{2}$ Structure Genomics Consortium Buchmann Institute for Molecular Life Sciences, Johann Wolfgang Goethe-University, Max-von-Laue-Str. 15, D-60438 Frankfurt afm Main, Germany.

*Correspondence: hanke@pharmchem.uni-frankfurt.de

knapp@pharmchem.uni-frankfurt.de

Supporting Information:

Contents:

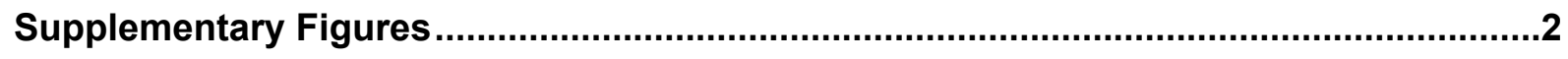

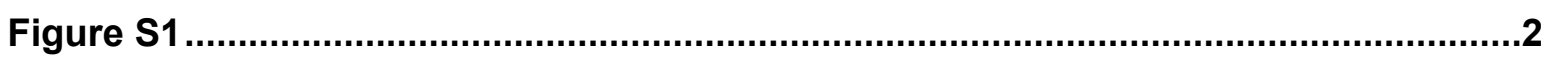

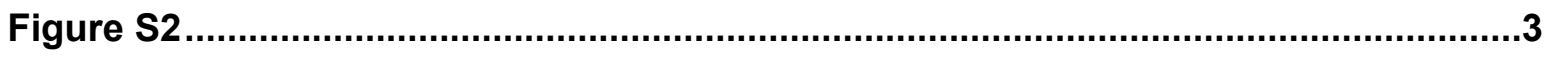

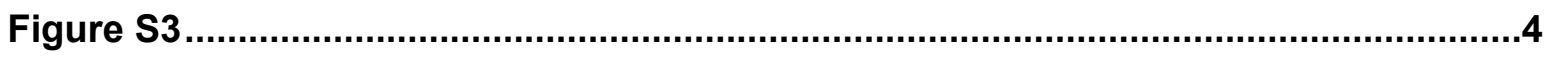

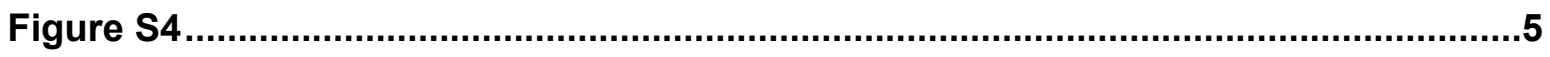

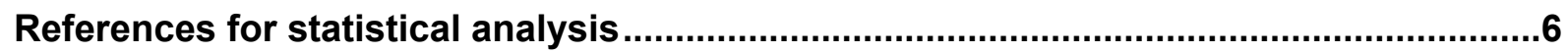




\section{Supplementary Figures}

\section{Figure S1}
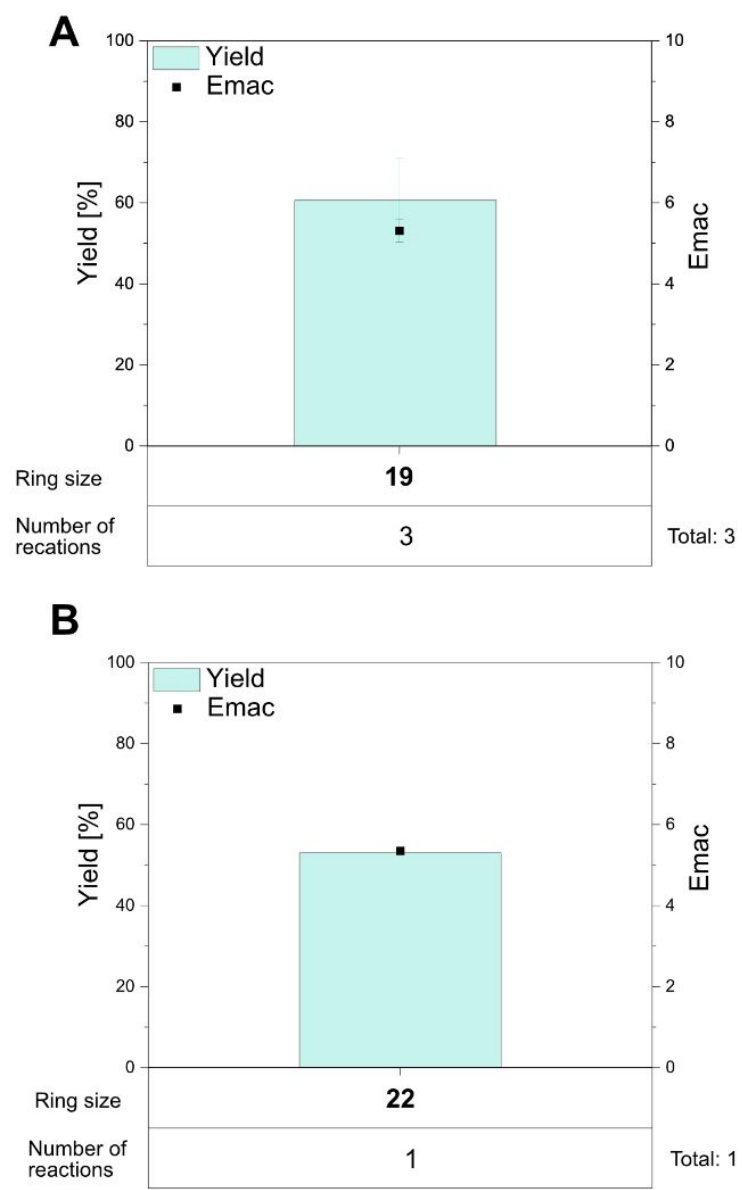

C

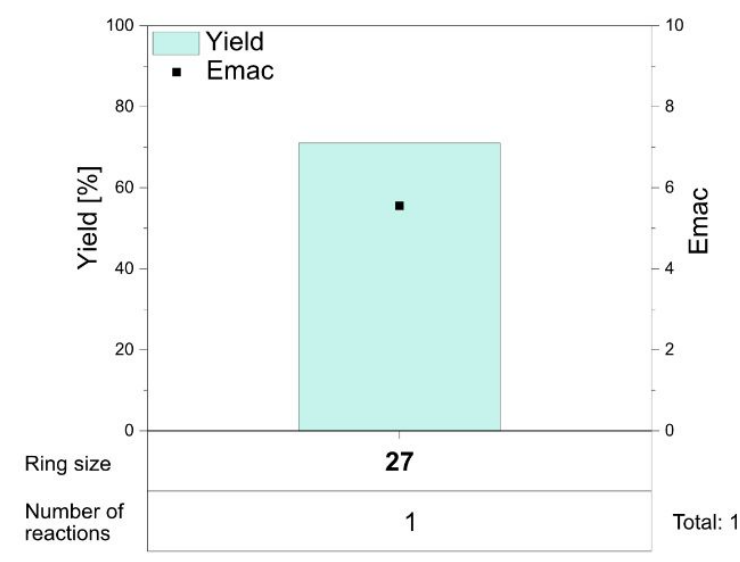

Figure S1: Summary of esterification ring closing reactions. Mean values of yields and Emac values depending on the ring size for the A: Mukaiyama reaction, B: Shiina esterfication, C: Steglich esterification. 


\section{Figure S2}
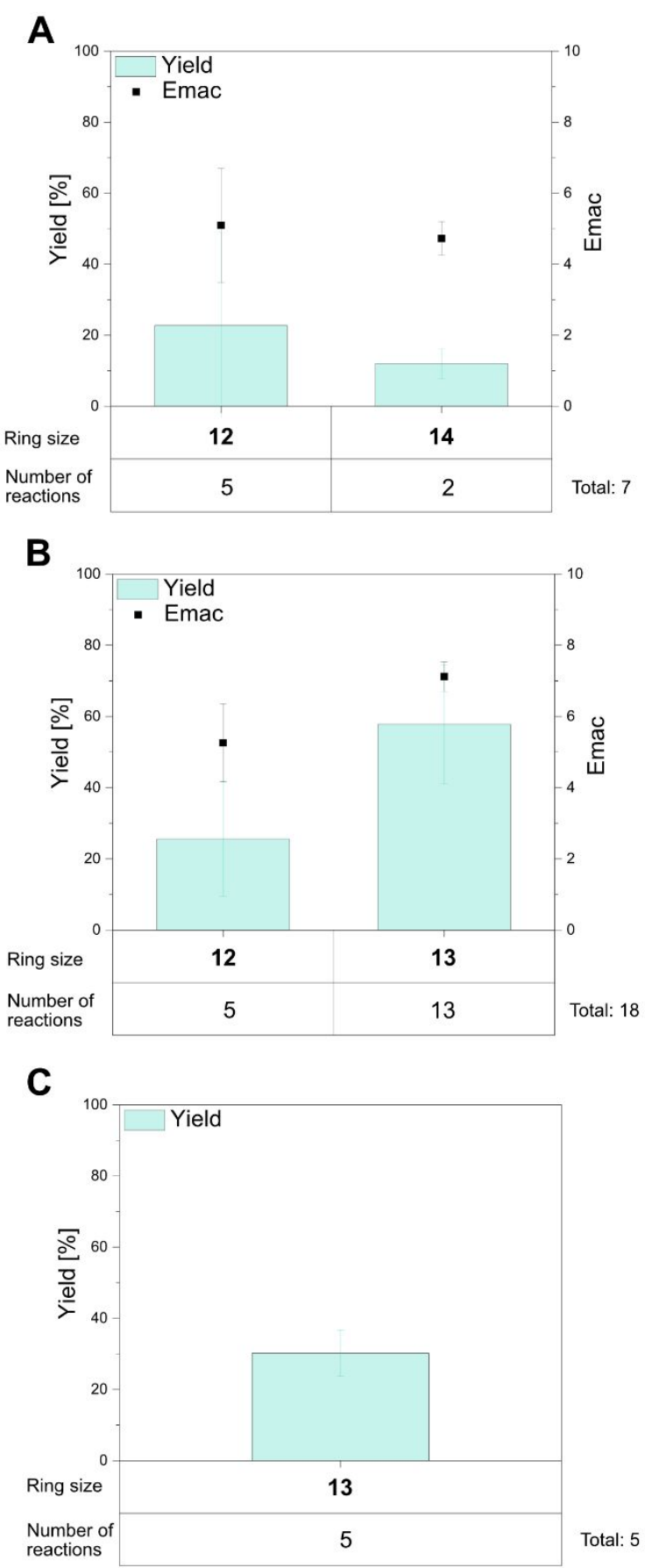

Figure S2: Summary of palladium-catalyzed cross-coupling reactions. Mean values of yields and Emac values depending on the ring size for the A: Suzuki reaction, B: Heck reaction, C: Buchwald-Hartwig amination. 


\section{Figure S3}

A

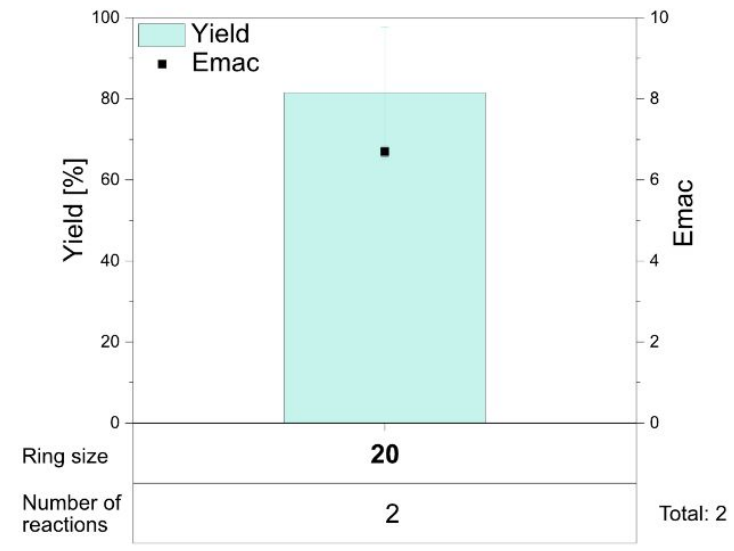

B

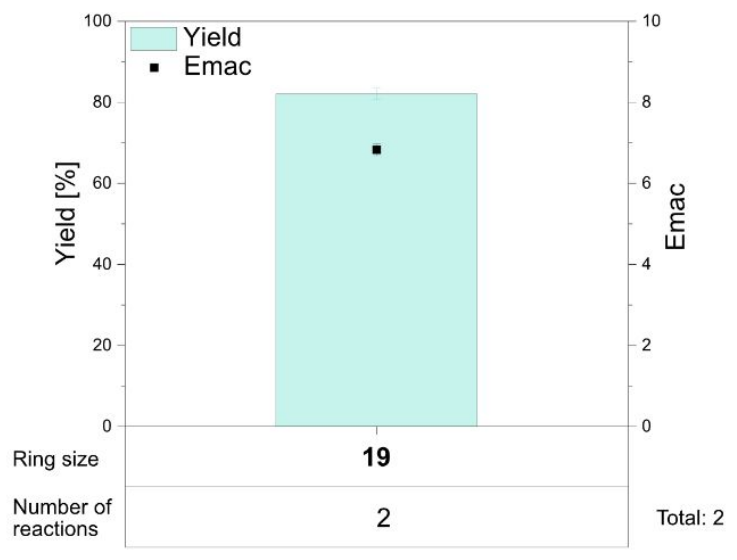

Figure S3: Summary of $\mathrm{C}-\mathrm{O}$ and $\mathrm{C}-\mathrm{N}$ bond formations. Mean values of yields and Emac values depending on the ring size for the A: Prins-driven reaction, B: Aryl amidation. 


\section{Figure S4}
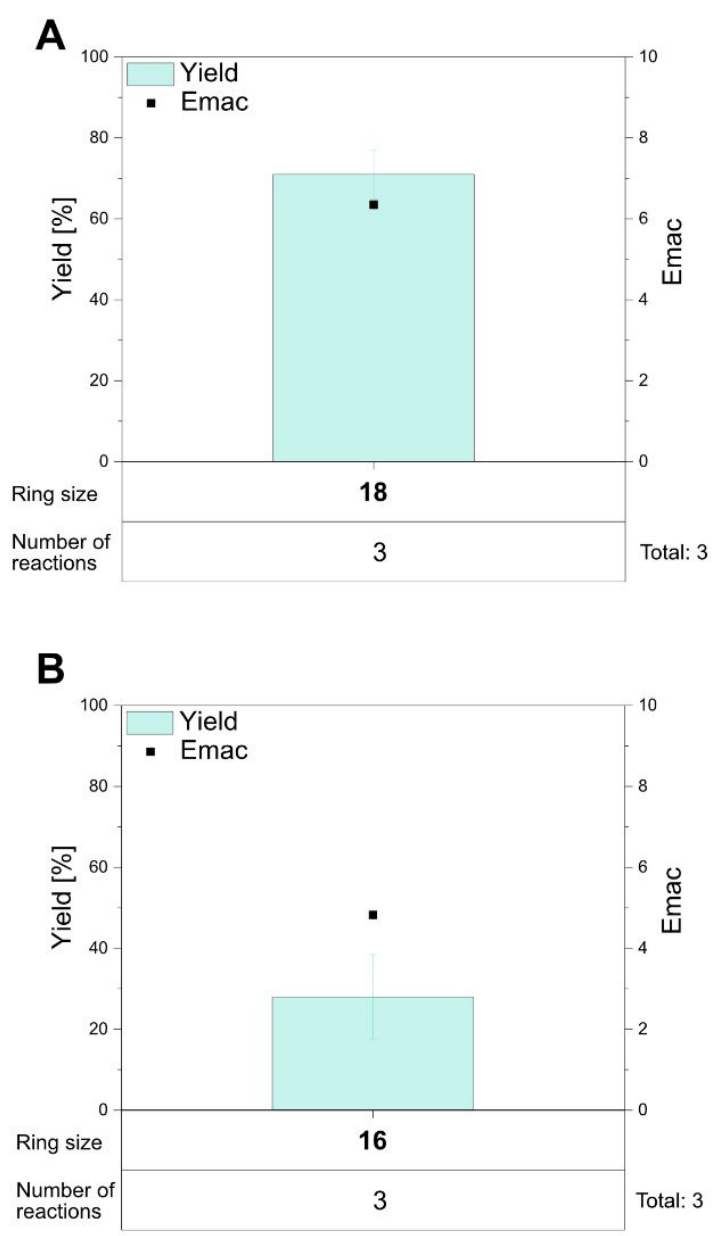

C

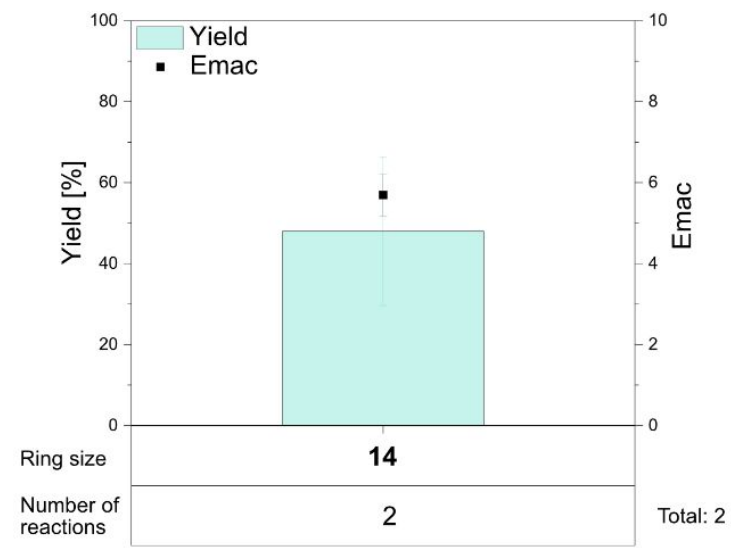

Figure S4: Summary of $\mathrm{C}-\mathrm{C}$ bond formations. Mean values of yields and Emac values depending on the ring size for the A: Wittig reaction, B: McMurry reaction, C: Nozaki-Hiyama-Kishi reaction. 


\section{References for statistical analysis}

The references for the 302 considered reactions are shown here. The different reaction types which were discribed in the article are the ring closing metathesis ${ }^{1-26}$, nucleophilic substitution 1,2,20,27-39, amide coupling 17,19,32,37,40-45, Yamaguchi esterfication ${ }^{3,46-53}$, Mukaiyama reaction $^{54}$, Shiina esterfication ${ }^{51}$, Steglich esterfication ${ }^{35}$, Suzuki reaction ${ }^{3,32}$, Heck reaction ${ }^{32,55,56}$, Buchwald-Hartwig amination ${ }^{57}$, Mitsunobu reaction ${ }^{3,34,41}$, Prins-driven reaction ${ }^{53,58}$, Click reaction ${ }^{30,59}$, aryl amidation ${ }^{60,61}$, alkyne metathesis ${ }^{62},[2+2+2]$ cycloaddition ${ }^{63,64}$, alkyne aldehyde reductive coupling ${ }^{65}$, Wittig reaction ${ }^{66-68}$, McMurry reaction $^{66,67}$ and Nozaki-Hiyama-Kishi reaction ${ }^{69}$.

References for the reactions, which could not be considered due to no yields have been reported, or the yield has been reported over several steps. The different reaction types which were discribed in the article are the ring closing metathesis ${ }^{12,19,21,37,8}$, nucleophilic substitution $^{2,13,32,34,70}$, amide coupling $4,19,32,37,71,72$, Yamaguchi esterfication ${ }^{73}$, Mukaiyama reaction ${ }^{3,39,74,75}$, Shiina esterfication ${ }^{51}$, Suzuki reaction ${ }^{19}$, Heck reaction ${ }^{32}$, Mitsunobu reaction $^{1,76},[2+2+2]$ cycloaddition ${ }^{64}$ and transannular aromatization ${ }^{77}$. 
(1) Dakas, P. Y.; Jogireddy, R.; Valot, G.; Barluenga, S.; Winssinger, N. Divergent Syntheses of Resorcylic Acid Lactones: L-783277, LL-Z1640-2, and Hypothemycin. Chem. - A Eur. J. 2009, 15 (43), 11490-11497.

(2) Cee, V. J.; Chavez, F.; Herberich, B.; Lanman, B. A.; Pettus, L. H.; Reed, A. B.; Wu, B.; Wurz, R. P.; Andrews, K. L.; Chen, J.; Hickman, D.; Laszlo, J.; Lee, M. R.; Guerrero, N.; Mattson, B. K.; Nguyen, Y.; Mohr, C.; Rex, K.; Sastri, C. E.; Wang, P.; Wu, Q.; Wu, T.; Xu, Y.; Zhou, Y.; Winston, J. T.; Lipford, J. R.; Tasker, A. S.; Wang, H. L. Discovery and Optimization of Macrocyclic Quinoxaline-Pyrrolo-

Dihydropiperidinones as Potent Pim-1/2 Kinase Inhibitors. ACS Med. Chem. Lett. 2016, 7 (4), 408-412.

(3) Hofmann, T.; Altmann, K. H. Resorcylic Acid Lactones as New Lead Structures for Kinase Inhibition. Comptes Rendus Chim. 2008, 11 (11-12), 1318-1335.

(4) Sasaki, Y.; Tokuhara, H.; Ohba, Y.; Okabe, A.; Nakayama, M.; Nakagawa, H.; Skene, R.; Hoffman, I.; Zou, H.; Yoshida, M. Efficient Synthesis of Tert-Butyl 3-Cyano-3Cyclopropyl-2-Oxopyrrolidine-4-Carboxylates: Highly Functionalized 2-Pyrrolidinone Enabling Access to Novel Macrocyclic Tyk2 Inhibitors. Bioorganic Med. Chem. Lett. 2020, 30 (5), 126963.

(5) Tao, Z. F.; Sowin, T. J.; Lin, N. H. Synthesis of Macrocyclic Urea Kinase Inhibitors. Synlett 2007, No. 18, 2855-2858.

(6) William, A. D.; Lee, A. C. H.; Blanchard, S.; Poulsen, A.; Teo, E. L.; Nagaraj, H.; Tan, E.; Chen, D.; Williams, M.; Sun, E. T.; Goh, K. C.; Ong, W. C.; Goh, S. K.; Hart, S.; Jayaraman, R.; Pasha, M. K.; Ethirajulu, K.; Wood, J. M.; Dymock, B. W. Discovery of the Macrocycle 11-(2-Pyrrolidin-1-YI-Ethoxy)-14,19-Dioxa-5,7, 26-Triaza-

Tetracyclo[19.3.1.1(2,6).1(8,12)] Heptacosa-1(25),2(26),3,5,8,10,12(27) ,16,21,23Decaene (SB1518), a Potent Janus Kinase 2/Fms-like Tyrosine Kinase-3 (JAK2/FLT3) Inhibito. J. Med. Chem. 2011, 54 (13), 4638-4658.

(7) Gunasekaran, P.; Lee, S. R.; Jeong, S. M.; Kwon, J. W.; Takei, T.; Asahina, Y.; Bang, G.; Kim, S.; Ahn, M.; Ryu, E. K.; Kim, H. N.; Nam, K. Y.; Shin, S. Y.; Hojo, H.;

Namgoong, S.; Kim, N. H.; Bang, J. K. Pyrrole-Based Macrocyclic Small-Molecule Inhibitors That Target Oocyte Maturation. ChemMedChem 2017, 12 (8), 580-589.

(8) William, A. D.; Lee, A. C. H.; Poulsen, A.; Goh, K. C.; Madan, B.; Hart, S.; Tan, E.; Wang, H.; Nagaraj, H.; Chen, D.; Lee, C. P.; Sun, E. T.; Jayaraman, R.; Pasha, M. K.; Ethirajulu, K.; Wood, J. M.; Dymock, B. W. Discovery of the Macrocycle (9 E)-15-(2(Pyrrolidin-1-YI)Ethoxy)-7,12,25- Trioxa-19,21,24-Triaza-

Tetracyclo[18.3.1.1(2,5).1(14,18)]Hexacosa-1(24),2,4,9, 14(26),15,17,20,22-Nonaene (SB1578), a Potent Inhibitor of Janus Kinase 2/Fms-Liketyrosine Kinase-3 (J. J. Med. Chem. 2012, 55 (6), 2623-2640.

(9) Thirupathi, B.; Mohapatra, D. K. First Total Synthesis of Cryptosporiopsin A. RSC Adv. 2014, 4 (16), 8027-8030.

(10) Tao, Z. F.; Chen, Z.; Bui, M. H.; Kovar, P.; Johnson, E.; Bouska, J.; Zhang, H.; Rosenberg, S.; Sowin, T.; Lin, N. H. Macrocyclic Ureas as Potent and Selective Chk1 Inhibitors: An Improved Synthesis, Kinome Profiling, Structure-Activity Relationships, and Preliminary Pharmacokinetics. Bioorganic Med. Chem. Lett. 2007, 17 (23), 65936601.

(11) Dutton, B. L.; Kitson, R. R. A.; Parry-Morris, S.; Roe, S. M.; Prodromou, C.; Moody, C. J. Synthesis of Macrolactam Analogues of Radicicol and Their Binding to Heat Shock Protein Hsp90. Org. Biomol. Chem. 2014, 12 (8), 1328-1340.

(12) Li, G.; Tao, Z. F.; Tong, Y.; Przytulinska, M. K.; Kovar, P.; Merta, P.; Chen, Z.; Zhang, H.; Sowin, T.; Rosenberg, S. H.; Lin, N. H. Synthesis and In-Vitro Biological Activity of 
Macrocyclic Urea Chk1 Inhibitors. Bioorganic Med. Chem. Lett. 2007, 17 (23), 64996504.

(13) McCoull, W.; Abrams, R. D.; Anderson, E.; Blades, K.; Barton, P.; Box, M.; Burgess, J.; Byth, K.; Cao, Q.; Chuaqui, C.; Carbajo, R. J.; Cheung, T.; Code, E.; Ferguson, A. D.; Fillery, S.; Fuller, N. O.; Gangl, E.; Gao, N.; Grist, M.; Hargreaves, D.; Howard, M. R.; Hu, J.; Kemmitt, P. D.; Nelson, J. E.; O'Connell, N.; Prince, D. B.; Raubo, P.; Rawlins, P. B.; Robb, G. R.; Shi, J.; Waring, M. J.; Whittaker, D.; Wylot, M.; Zhu, X. Discovery of Pyrazolo[1,5-a]Pyrimidine B-Cell Lymphoma 6 (BCL6) Binders and Optimization to High Affinity Macrocyclic Inhibitors. J. Med. Chem. 2017, 60 (10), 4386-4402.

(14) Hanessian, S.; Szychowski, J.; Campos-Reales Pineda, N. B.; Furtos, A.; Keillor, J. W. 6-Hydroxy to 6'--Amino Tethered Ring-to-Ring Macrocyclic Aminoglycosides as Probes for APH(3')-Illa Kinase. Bioorganic Med. Chem. Lett. 2007, 17 (11), 3221-3225.

(15) Barluenga, S.; Dakas, P. Y.; Ferandin, Y.; Meijer, L.; Winssinger, N. Modular Asymmetric Synthesis of Aigialomycin D, a Kinase-Inhibitory Scaffold. Angew. Chemie - Int. Ed. 2006, 45 (24), 3951-3954.

(16) Matos, M. C.; Murphy, P. V. Synthesis of Macrolide-Saccharide Hybrids by RingClosing Metathesis of Precursors Derived from Glycitols and Benzoic Acids. J. Org. Chem. 2007, 72 (5), 1803-1806.

(17) Himmelbauer, M. K.; Xin, Z.; Jones, J. H.; Enyedy, I.; King, K.; Marcotte, D. J.; Murugan, P.; Santoro, J. C.; Hesson, T.; Spilker, K.; Johnson, J. L.; Luzzio, M. J.; Gilfillan, R.; De Turiso, F. G. L. Rational Design and Optimization of a Novel Class of Macrocyclic Apoptosis Signal-Regulating Kinase 1 Inhibitors. J. Med. Chem. 2019, 62 (23), 10740-10756.

(18) Ning, C. Q.; Lu, C.; Hu, L.; Bi, Y. J.; Yao, L.; He, Y. J.; Liu, L. F.; Liu, X. Y.; Yu, N. F. Macrocyclic Compounds as Anti-Cancer Agents: Design and Synthesis of Multi-Acting Inhibitors against HDAC, FLT3 and JAK2. Eur. J. Med. Chem. 2015, 95, 104-115.

(19) Wang, X.; Liu, J.; Zhang, W.; Stashko, M. A.; Nichols, J.; Miley, M. J.; Norris-Drouin, J.; Chen, Z.; Machius, M.; DeRyckere, D.; Wood, E.; Graham, D. K.; Earp, H. S.; Kireev, D.; Frye, S. V. Design and Synthesis of Novel Macrocyclic Mer Tyrosine Kinase Inhibitors. ACS Med. Chem. Lett. 2016, 7 (12), 1044-1049.

(20) Xu, J.; Chen, A.; Joy, J.; Xavier, V. J.; Ong, E. H. Q.; Hill, J.; Chai, C. L. L. Rational Design of Resorcylic Acid Lactone Analogues as Covalent MNK1/2 Kinase Inhibitors by Tuning the Reactivity of an Enamide Michael Acceptor. ChemMedChem 2013, 8 (9), 1483-1494.

(21) Tao, Z. F.; Wang, L.; Stewart, K. D.; Chen, Z.; Gu, W.; Bui, M. H.; Merta, P.; Zhang, H.; Kovar, P.; Johnson, E.; Park, C.; Judge, R.; Rosenberg, S.; Sowin, T.; Lin, N. H. Structure-Based Design, Synthesis, and Biological Evaluation of Potent and Selective Macrocyclic Checkpoint Kinase 1 Inhibitors. J. Med. Chem. 2007, 50 (7), 1514-1527.

(22) McErlean, C. S. P.; Proisy, N.; Davis, C. J.; Boland, N. A.; Sharp, S. Y.; Boxall, K.; Slawin, A. M. Z.; Workman, P.; Moody, C. J. Synthetic Ansamycins Prepared by a Ring-Expanding Claisen Rearrangement. Synthesis and Biological Evaluation of Ring and Conformational Analogues of the Hsp90 Molecular Chaperone Inhibitor Geldanamycin. Org. Biomol. Chem. 2007, 5 (3), 531-546.

(23) Day, J. E. H.; Sharp, S. Y.; Rowlands, M. G.; Aherne, W.; Hayes, A.; Raynaud, F. I.; Lewis, W.; Roe, S. M.; Prodromou, C.; Pearl, L. H.; Workman, P.; Moody, C. J. Targeting the Hsp90 Molecular Chaperone with Novel Macrolactams. Synthesis, Structural, Binding, and Cellular Studies. ACS Chem. Biol. 2011, 6 (12), 1339-1347. 
(24) Fuse, S.; Sugiyama, S.; Takahashi, T. Rapid Assembly of Resorcylic Acid Lactone Frameworks through Sequential Palladium-Catalyzed Coupling Reactions. Chem. - An Asian J. 2010, 5 (12), 2459-2462.

(25) Barluenga, S.; Moulin, E.; Lopez, P.; Winssinger, N. Solution- and Solid-Phase Synthesis of Radicicol (Monorden) and Pochonin C. Chem. - A Eur. J. 2005, 11 (17), 4935-4952.

(26) William, A. D.; Lee, A. C. H.; Goh, K. C.; Blanchard, S.; Poulsen, A.; Teo, E. L.; Nagaraj, H.; Lee, C. P.; Wang, H.; Williams, M.; Sun, E. T.; Hu, C.; Jayaraman, R.; Pasha, M. K.; Ethirajulu, K.; Wood, J. M.; Dymock, B. W. Discovery of Kinase Spectrum Selective Macrocycle (16E)-14-Methyl-20-Oxa-5, 7,14,26Tetraazatetracyclo[19.3.1.1(2,6).1(8,12)]Heptacosa-1(25),2(26),3,5,8(27) ,9,11,16,21,23-Decaene (SB1317/TG02), a Potent Inhibitor of Cyclin Dependent Kinases (CDKs), Janus. J. Med. Chem. 2012, 55 (1), 169-196.

(27) Gómez-Pérez, V.; Manzano, J. I.; García-Hernández, R.; Castanys, S.; Gamarro, F.; Campos, J. M. Design, Synthesis and Anti-Leishmanial Activity of Novel Symmetrical Bispyridinium Cyclophanes. Eur. J. Med. Chem. 2015, 89, 362-369.

(28) Lewin, A. H.; Brieaddy, L.; Deschamps, J. R.; Imler, G. H.; Mascarella, S. W.; Reddy, P. A.; Carroll, F. I. Synthesis and Characterization of the Selective, Reversible PKC $\beta$ Inhibitor (9 S)-9-[(Dimethylamino)Methyl]-6,7,10,11-Tetrahydro-9 H,18 H-5,21:12,17Dimethenodibenzo[ e,k]Pyrrolo[3,4- h][1,4,13]Oxadiazacyclohexadecine-18,20(19 H)Dione, Ruboxistaurin (LY3. ACS Chem. Neurosci. 2019, 10 (1), 246-251.

(29) Rotger, C.; Piña, M. N.; Vega, M.; Ballester, P.; Deyà, P. M.; Costa, A. Efficient Macrocyclization of Preorganized Palindromic Oligosquaramides. Angew. Chemie Int. Ed. 2006, 45 (41), 6844-6848.

(30) Goh, W. Y. L.; Chai, C. L. L.; Chen, A. Synthesis and Biological Studies of a Triazole Analogue of Resorcylic Acid Lactone LL-Z1640-2. European J. Org. Chem. 2014, 2014 (32), 7239-7244.

(31) Maddess, M. L.; Tackett, M. N.; Watanabe, H.; Brennan, P. E.; Spilling, C. D.; Scott, J. S.; Osborn, D. P.; Ley, S. V. Total Synthesis of Rapamycin. Angew. Chemie - Int. Ed. 2007, 46 (4), 591-597.

Johnson, T. W.; Richardson, P. F.; Bailey, S.; Brooun, A.; Burke, B. J.; Collins, M. R.; Cui, J. J.; Deal, J. G.; Deng, Y. L.; Dinh, D.; Engstrom, L. D.; He, M.; Hoffman, J.; Hoffman, R. L.; Huang, Q.; Kania, R. S.; Kath, J. C.; Lam, H.; Lam, J. L.; Le, P. T.; Lingardo, L.; Liu, W.; McTigue, M.; Palmer, C. L.; Sach, N. W.; Smeal, T.; Smith, G. L.; Stewart, A. E.; Timofeevski, S.; Zhu, H.; Zhu, J.; Zou, H. Y.; Edwards, M. P. Discovery of (10 R)-7-Amino-12-Fluoro-2,10,16-Trimethyl-15-Oxo-10,15,16,17- Tetrahydro- 2H 8,4-(Metheno)Pyrazolo[4,3- $\mathrm{h}$ ][2,5,11]- Benzoxadiazacyclotetradecine-3-Carbonitrile (PF-06463922), a Macrocyclic Inhibitor of Anaplastic Lymphoma Kinase (ALK) And. J. Med. Chem. 2014, 57 (11), 4720-4744.

(33) Fedorov, O.; Marsden, B.; Pogacic, V.; Rellos, P.; Müller, S.; Bullock, A. N.; Schwaller, J.; Sundström, M.; Knapp, S. A Systematic Interaction Map of Validated Kinase Inhibitors with Ser/Thr Kinases. Proc. Natl. Acad. Sci. U. S. A. 2007, 104 (51), $20523-$ 20528.

(34) Lücking, U.; Siemeister, G.; Schäfer, M.; Briem, H.; Krüger, M.; Lienau, P.; Jautelat, R. Macrocyclic Aminopyrimidines as Multitarget CDK and VEGF-R Inhibitors with Potent Antiproliferative Activities. ChemMedChem 2007, 2 (1), 63-77.

(35) Ley, S. V.; Tackett, M. N.; Maddess, M. L.; Anderson, J. C.; Brennan, P. E.; Cappi, M. W.; Heer, J. P.; Helgen, C.; Kori, M.; Kouklovsky, C.; Marsden, S. P.; Norman, J.;

Osborn, D. P.; Palomero, M. Á.; Pavey, J. B. J.; Pinel, C.; Robinson, L. A.; Schnaubelt, 
J.; Scott, J. S.; Spilling, C. D.; Watanabe, H.; Wesson, K. E.; Willis, M. C. Total Synthesis of Rapamycin. Chem. - A Eur. J. 2009, 15 (12), 2874-2914.

(36) Katoh, T.; Tomata, Y.; Setoh, M.; Sasaki, S.; Takai, T.; Yoshitomi, Y.; Yukawa, T.; Nakagawa, H.; Fukumoto, S.; Tsukamoto, T.; Nakada, Y. Practical Application of 3Substituted-2,6-Difluoropyridines in Drug Discovery: Facile Synthesis of Novel Protein Kinase C Theta Inhibitors. Bioorganic Med. Chem. Lett. 2017, 27 (11), 2497-2501.

(37) Mclver, A. L.; Zhang, W.; Liu, Q.; Jiang, X.; Stashko, M. A.; Nichols, J.; Miley, M. J.; Norris-Drouin, J.; Machius, M.; DeRyckere, D.; Wood, E.; Graham, D. K.; Earp, H. S.; Kireev, D.; Frye, S. V.; Wang, X. Discovery of Macrocyclic Pyrimidines as MerTKSpecific Inhibitors. ChemMedChem 2017, 12 (3), 207-213.

(38) Villalonga, P.; FernándezdeMattos, S.; Ramis, G.; Obrador-Hevia, A.; Sampedro, A.; Rotger, C.; Costa, A. Cyclosquaramides as Kinase Inhibitors with Anticancer Activity. ChemMedChem 2012, 7 (8), 1472-1480.

(39) Shen, Y.; Boivin, R.; Yoneda, N.; Du, H.; Schiller, S.; Matsushima, T.; Goto, M.; Shirota, H.; Gusovsky, F.; Lemelin, C.; Jiang, Y.; Zhang, Z.; Pelletier, R.; IkemoriKawada, M.; Kawakami, Y.; Inoue, A.; Schnaderbeck, M.; Wang, Y. Discovery of AntiInflammatory Clinical Candidate E6201, Inspired from Resorcylic Lactone LL-Z1640-2, III. Bioorganic Med. Chem. Lett. 2010, 20 (10), 3155-3157.

(40) Canova, S.; Bellosta, V.; Bigot, A.; Mailliet, P.; Mignani, S.; Cossy, J. Total Synthesis of Herbimycin A. Org. Lett. 2007, 9 (1), 145-148.

(41) Engelhardt, H.; Böse, D.; Petronczki, M.; Scharn, D.; Bader, G.; Baum, A.; Bergner, A.; Chong, E.; Döbel, S.; Egger, G.; Engelhardt, C.; Ettmayer, P.; Fuchs, J. E.; Gerstberger, T.; Gonnella, N.; Grimm, A.; Grondal, E.; Haddad, N.; Hopfgartner, B.; Kousek, R.; Krawiec, M.; Kriz, M.; Lamarre, L.; Leung, J.; Mayer, M.; Patel, N. D.; Simov, B. P.; Reeves, J. T.; Schnitzer, R.; Schrenk, A.; Sharps, B.; Solca, F.; Stadtmüller, H.; Tan, Z.; Wunberg, T.; Zoephel, A.; McConnell, D. B. Start Selective and Rigidify: The Discovery Path toward a Next Generation of EGFR Tyrosine Kinase Inhibitors. J. Med. Chem. 2019, 62 (22), 10272-10293.

(42) Farand, J.; Mai, N.; Chandrasekhar, J.; Newby, Z. E.; Van Veldhuizen, J.; Loyer-Drew, J.; Venkataramani, C.; Guerrero, J.; Kwok, A.; Li, N.; Zherebina, Y.; Wilbert, S.; Zablocki, J.; Phillips, G.; Watkins, W. J.; Mourey, R.; Notte, G. T. Selectivity Switch between FAK and Pyk2: Macrocyclization of FAK Inhibitors Improves Pyk2 Potency. Bioorganic Med. Chem. Lett. 2016, 26 (24), 5926-5930.

(43) Belardi, J. K.; Micalizio, G. C. Total Synthesis of Macbecin I. Angew. Chemie - Int. Ed. 2008, 47 (21), 4005-4008. https://doi.org/10.1002/anie.200800400.

(44) Suda, A.; Koyano, H.; Hayase, T.; Hada, K.; Kawasaki, K. I.; Komiyama, S.; Hasegawa, K.; Fukami, T. A.; Sato, S.; Miura, T.; Ono, N.; Yamazaki, T.; Saitoh, R.; Shimma, N.; Shiratori, Y.; Tsukuda, T. Design and Synthesis of Novel Macrocyclic 2Amino-6-Arylpyrimidine Hsp90 Inhibitors. Bioorganic Med. Chem. Lett. 2012, 22 (2), $1136-1141$.

(45) Yan, R.; Bian, C.; Yu, X. Total Synthesis of Herbimycin A. Org. Lett. 2014, 16 (12), 3280-3283.

(46) Baumann, D. O.; McGowan, K. M.; Kedei, N.; Peach, M. L.; Blumberg, P. M.; Keck, G. E. Synthesis and Biological Evaluation of Several Bryostatin Analogues Bearing a Diacylglycerol Lactone C-Ring. J. Org. Chem. 2016, 81 (17), 7862-7883.

(47) Zhang, Y.; Guo, Q.; Sun, X.; Lu, J.; Cao, Y.; Pu, Q.; Chu, Z.; Gao, L.; Song, Z. Total Synthesis of Bryostatin 8 Using an Organosilane-Based Strategy. Angew. Chemie Int. Ed. 2018, 57 (4), 942-946. 
(48) Kraft, M. B.; Poudel, Y. B.; Kedei, N.; Lewin, N. E.; Peach, M. L.; Blumberg, P. M.; Keck, G. E. Synthesis of a Des-B-Ring Bryostatin Analogue Leads to an Unexpected Ring Expansion of the Bryolactone Core. J. Am. Chem. Soc. 2014, 136 (38), 1320213208.

(49) Keck, G. E.; Poudel, Y. B.; Cummins, T. J.; Rudra, A.; Covel, J. A. Total Synthesis of Bryostatin 1. J. Am. Chem. Soc. 2011, 133 (4), 744-747.

(50) Lu, J.; Ma, J.; Xie, X.; Chen, B.; She, X.; Pan, X. Enantioselective Total Synthesis of Aigialomycin D. Tetrahedron Asymmetry 2006, 17 (7), 1066-1073.

(51) Ketcham, J. M.; Volchkov, I.; Chen, T. Y.; Blumberg, P. M.; Kedei, N.; Lewin, N. E.; Krische, M. J. Evaluation of Chromane-Based Bryostatin Analogues Prepared via Hydrogen-Mediated C-C Bond Formation: Potency Does Not Confer Bryostatin-like Biology. J. Am. Chem. Soc. 2016, 138 (40), 13415-13423.

(52) Wender, P. A.; Hardman, C. T.; Ho, S.; Jeffreys, M. S.; Maclaren, J. K.; Quiroz, R. V.; Ryckbosch, S. M.; Shimizu, A. J.; Sloane, J. L.; Stevens, M. C. Scalable Synthesis of Bryostatin 1 and Analogs, Adjuvant Leads against Latent HIV. Science (80-. ). 2017, 358 (6360), 218-223.

(53) Cummins, T. J.; Kedei, N.; Czikora, A.; Lewin, N. E.; Kirk, S.; Petersen, M. E.; McGowan, K. M.; Chen, J. Q.; Luo, X.; Johnson, R. C.; Ravichandran, S.; Blumberg, P. M.; Keck, G. E. Synthesis and Biological Evaluation of Fluorescent Bryostatin Analogues. ChemBioChem 2018, 19 (8), 877-889.

(54) Jeso, V.; Iqbal, S.; Hernandez, P.; Cameron, M. D.; Park, H.; Lograsso, P. V.; Micalizio, G. C. Synthesis of Benzoquinone Ansamycin-Inspired Macrocyclic Lactams from Shikimic Acid. Angew. Chemie - Int. Ed. 2013, 52 (18), 4800-4804.

(55) Basit, S.; Ashraf, Z.; Lee, K.; Latif, M. First Macrocyclic 3rd-Generation ALK Inhibitor for Treatment of ALK/ROS1 Cancer: Clinical and Designing Strategy Update of Lorlatinib. Eur. J. Med. Chem. 2017, 134, 348-356.

(56) Breslin, H. J.; Lane, B. M.; Ott, G. R.; Ghose, A. K.; Angeles, T. S.; Albom, M. S.; Cheng, M.; Wan, W.; Haltiwanger, R. C.; Wells-Knecht, K. J.; Dorsey, B. D. Design, Synthesis, and Anaplastic Lymphoma Kinase (ALK) Inhibitory Activity for a Novel Series of 2,4,8,22-Tetraazatetracyclo[14.3.1.1 3,7.1 9,13]Docosa1(20),3(22),4,6,9(21), 10,12,16,18-Nonaene Macrocycles. J. Med. Chem. 2012, 55 (1), 449-464.

(57) Wang, Y.; Ge, H.; Wang, D.; He, H.; Li, L.; Diao, Y.; Shen, Z.; Zhu, L.; Li, S.; Zhao, Z.; $\mathrm{Li}, \mathrm{H}$. Design and Synthesis of a Series of Novel Macrocycle Janus Kinase 2 Inhibitors. Chinese J. Chem. 2019, 37 (12), 1259-1263.

(58) Wender, P. A.; DeChristopher, B. A.; Schrier, A. J. Efficient Synthetic Access to a New Family of Highly Potent Bryostatin Analogues via a Prins-Driven Macrocyclization Strategy. J. Am. Chem. Soc. 2008, 130 (21), 6658-6659.

(59) Cruz-López, O.; Temps, C.; Longo, B.; Myers, S. H.; Franco-Montalban, F.; UncitiBroceta, A. Synthesis and Characterization of a Click-Assembled 18-Atom Macrocycle That Displays Selective AXL Kinase Inhibitory Activity. ACS Omega 2019, 4 (25), 21620-21626.

(60) Wrona, I. E.; Gabarda, A. E.; Evano, G.; Panek, J. S. Total Synthesis of Reblastatin. J. Am. Chem. Soc. 2005, 127 (43), 15026-15027.

(61) Qin, H.-L.; Panek, J. S. Total Synthesis of the Hsp90 Inhibitor Geldanamycin. Org. Lett. 2008, 10 (12), 2477-2479.

(62) Schaubach, S.; Gebauer, K.; Ungeheuer, F.; Hoffmeister, L.; Ilg, M. K.; Wirtz, C.; 
Fürstner, A. A Two-Component Alkyne Metathesis Catalyst System with an Improved Substrate Scope and Functional Group Tolerance: Development and Applications to Natural Product Synthesis. Chem. - A Eur. J. 2016, 22 (25), 8494-8507.

(63) Maryanoff, B. E.; Zhang, H. Pyridine-Containing Macrocycles via Cobalt-Mediated [2 + 2 + 2] Cycloadditions of $\alpha, \omega$-Bis-Alkynes. ARKIVOC 2007, 2007 (12), 7-35.

(64) Zhang, H. C.; Boñaga, L. V. R.; Ye, H.; Derian, C. K.; Damiano, B. P.; Maryanoff, B. E. Novel Bis(Indolyl)Maleimide Pyridinophanes That Are Potent, Selective Inhibitors of Glycogen Synthase Kinase-3. Bioorganic Med. Chem. Lett. 2007, 17 (10), 2863-2868.

(65) Wang, S. Q.; Goh, S. S.; Chai, C. L. L.; Chen, A. An Efficient Synthesis of an ExoEnone Analogue of LL-Z1640-2 and Evaluation of Its Protein Kinase Inhibitory Activities. Org. Biomol. Chem. 2016, 14 (2), 639-645.

(66) Jiang, J.; Sun, B.; Wang, Y. Y.; Cui, M.; Zhang, L.; Cui, C. Z.; Wang, Y. F.; Liu, X. G.; Lou, H. X. Synthesis of Macrocyclic Bisbibenzyl Derivatives and Their Anticancer Effects as Anti-Tubulin Agents. Bioorganic Med. Chem. 2012, 20 (7), 2382-2391.

(67) Speicher, A.; Groh, M.; Zapp, J.; Schaumlöffel, A.; Knauer, M.; Bringmann, G. A Synthesis-Driven Structure Revision of "Plagiochin E", a Highly Bioactive Bisbibenzyl. Synlett 2009, No. 11, 1852-1858.

(68) Sun, B.; Yuan, H. qing; Xi, G. min; Ma, Y. dao; Lou, H. xiang. Synthesis and Multidrug Resistance Reversal Activity of Dihydroptychantol A and Its Novel Derivatives. Bioorganic Med. Chem. 2009, 17 (14), 4981-4989.

(69) LeClair, C. A.; Boxer, M. B.; Thomas, C. J.; Maloney, D. J. Total Synthesis of LLZ1640-2 Utilizing a Late-Stage Intramolecular Nozaki-Hiyama-Kishi Reaction. Tetrahedron Lett. 2010, 51 (52), 6852-6855.

(70) Kawanishi, N.; Sugimoto, T.; Shibata, J.; Nakamura, K.; Masutani, K.; Ikuta, M.; Hirai, H. Structure-Based Drug Design of a Highly Potent CDK1,2,4,6 Inhibitor with Novel Macrocyclic Quinoxalin-2-One Structure. Bioorganic Med. Chem. Lett. 2006, 16 (19), 5122-5126.

(71) Shen, G.; Wang, M.; Welch, T. R.; Blagg, B. S. J. Design, Synthesis, and Structure Activity Relationships for Chimeric Inhibitors of Hsp90. J. Org. Chem. 2006, 71 (20), 7618-7631.

(72) Napolitano, C.; Palwai, V. R.; Eriksson, L. A.; Murphy, P. V. Synthesis, Kinase Activity and Molecular Modeling of a Resorcylic Acid Lactone Incorporating an Amide and a Trans-Enone in the Macrocycle. Tetrahedron 2012, 68 (27-28), 5533-5540.

(73) Zhao, X.; Kedei, N.; Michalowski, A.; Lewin, N. E.; Keck, G. E.; Blumberg, P. M. Deletion of the C26 Methyl Substituent from the Bryostatin Analogue Merle 23 Has Negligible Impact on Its Biological Profile and Potency. ChemBioChem 2018, 19 (10), 1049-1059.

(74) Kashin, D.; Meyer, A.; Wittenberg, R.; Schöning, K. U.; Kamlage, S.; Kirschning, A. A Practical Synthesis of the Ansa Chain of Benzenic Ansamycin Antibiotics: Total Synthesis of an Ansatrienol Derivative. Synthesis (Stuttg). 2007, No. 2, 304-319.

(75) Zhuo, C. X.; Fürstner, A. Catalysis-Based Total Syntheses of Pateamine A and DMDA-Pat A. J. Am. Chem. Soc. 2018, 140 (33), 10514-10523.

(76) Wender, P. A.; Nakagawa, Y.; Near, K. E.; Staveness, D. Computer-Guided Design, Synthesis, and Protein Kinase C Affinity of a New Salicylate-Based Class of Bryostatin Analogs. Org. Lett. 2014, 16 (19), 5136-5139.

(77) Miyatake-Ondozabal, H.; Barrett, A. G. M. Total Synthesis of TAK-Kinase Inhibitor LL- 
Z1640-2 via Consecutive Macrocyclization and Transannular Aromatization. Org. Lett. 2010, 12 (23), 5573-5575. 